\title{
A NOTE ON GRASHOF THEOREM
}

Wen-Tung Chang

Ph.D. Candidate of Power Mechanical Engineering, National Tsing Hua University, Hsinchu, Taiwan 30013, R.O.C

Chen-Chou Lin

Professor of Mechanical and Mechatronic Engineering, National Taiwan Ocean University, Keelung, Taiwan 20224,

R.O.C, cclin@euler.me.ntou.edu.tw

Long-long Wu

Professor of Power Mechanical Engineering, National Tsing Hua University, Hsinchu, Taiwan 30013, R.O.C

Follow this and additional works at: https://jmstt.ntou.edu.tw/journal

Part of the Mechanical Engineering Commons

\section{Recommended Citation}

Chang, Wen-Tung; Lin, Chen-Chou; and Wu, Long-long (2005) "A NOTE ON GRASHOF THEOREM," Journal of Marine Science and Technology. Vol. 13: Iss. 4, Article 1.

DOI: $10.51400 / 2709-6998.2117$

Available at: https://jmstt.ntou.edu.tw/journal/vol13/iss4/1

This Research Article is brought to you for free and open access by Journal of Marine Science and Technology. It has been accepted for inclusion in Journal of Marine Science and Technology by an authorized editor of Journal of Marine Science and Technology. 


\title{
A NOTE ON GRASHOF'S THEOREM
}

\author{
Wen-Tung Chang*, Chen-Chou Lin**, and Long-Iong Wu***
}

Key words: Grashof's theorem, stationary configuration, uncertainty configuration, planar four-bar linkage.

\section{ABSTRACT}

In this paper, Grashof's theorem is justified from the perspective of the occurrence of stationary configurations and uncertainty configurations of four-link chains. The link length relations at stationary configurations and uncertainty configurations are examined in detail based on the triangle inequality, and then the conditions for the existence of a crank and a change point are deduced.

\section{INTRODUCTION}

The mobility problem has been of interest for a long time in the area of mechanism design. Grashof [7] first introduced a very simple rule to judge the rotatability of links in four-bar linkages. Although the problem drew the attention of many researchers $[4,8,9]$, the rule, known as Grashof's theorem today, was not formally proved until Paul [13] published his paper. In that paper, Paul applied the triangle inequality to analyze the mobility of four-link chains under their limit positions, and proved that Grashof's criterion is a necessary and sufficient condition for the existence of a crank in a four-link chain. He also proved the equality form of Grashof's criterion is a necessary and sufficient condition for the existence of a change point mechanism. After the reassessment of Grashof's criterion by Paul, many works about the mobility of planar four-bar linkages were undertaken. Angeles and Callejas [1] derived the constraining inequalities of displacement functions of four-bar linkages, based on Freudenstein's equation [6]. The constraints, equivalent to Grashof's criteria, were applied to the optimal mobility design problems. Midha et al. [12] utilized the triangle inequality concept

Paper Submitted 03/05/05, Accepted 06/23/05. Author for Correspondence: Chen-Chou Lin. E-mail: cclin@euler.me.ntou.edu.tw.

*Ph.D. Candidate of Power Mechanical Engineering, National Tsing Hua University, Hsinchu, Taiwan 30013, R.O.C.

**Professor of Mechanical and Mechatronic Engineering, National Taiwan Ocean University, Keelung, Taiwan 20224, R.O.C.

***Professor of Power Mechanical Engineering, National Tsing Hua University, Hsinchu, Taiwan 30013, R.O.C. to formulate the mobility conditions for planar four-bar linkages, and provided a graphical interpretation to mobility determination. The concept can be extended to a more complex planar linkage. Barker [3] classified planar four-bar linkages into four classes of Grashof's category, four classes of non-Grashof's category, and six classes of change point category, based on the properties of characteristic surfaces in positive octant regions. Cohan and Yang [5] developed a transformation between Euclidean space and "parallel coordinates system", and analysed the mobility of linkages by the ranges of the angular coordinates. Williams and Reinholtz [18] proved that the polynomial discriminant of four-bar linkages, after reductions, is equivalent to Grashof's law. Besides the mobility analysis of planar four-bar linkages, some works were focused on the areas of linkages with more than four bars and spatial linkages. Ting [15] first introduced the Five-bar Grashof's criteria. The mobility criteria were further extended to N-bar kinematic chains later by Ting [16] and Ting and Liu [17]. In their derivations of rotatability laws for N-bar linkages, Grashof's theorem became a very special case.

A mechanism may encounter a singular configuration under certain geometric conditions. At such conditions, the instantaneous degree-of-freedom (d.o. f.) or the transitory mobility of the mechanism will be different from the result derived by the Grübler's equation. When a singular configuration occurs during the motion, it represents that the relative motion state in the mechanism is in a critical state; usually, the mobility of some link (s) will become zero. Thus a study of the geometric relations for linkages under singular conditions may help in understanding the mobility of the linkages. Singular configurations can be further categorized into [19]: (a) stationary configuration, (b) uncertainty configuration, and (c) dead-center configuration.

The main objective of this paper is to provide an alternative proof to Grashof's theorem from the perspective of the occurrence of singular configurations. The geometric relations of four-link chains at stationary configurations and uncertainty configurations are examined in detail, and then the conditions for the existence of a crank and a change point are deduced. 


\section{GRASHOF'S THEOREM}

Grashof's theorem [7] was first introduced in 1883 to deal with the mobility problem of planar four-bar mechanisms, in particular, mechanisms with all revolute joints. Consider a four-link kinematic chain, let $s$ be the shortest link length, $l$ be the longest link length, $p$ and $q$ be the link lengths of the remaining two links, where $1>p \geq q>s$. Grashof stated that there exists at least one link which can fully revolve with respect to the other three links if

$$
1+s \leq p+q
$$

and none of the four links can make a full revolution if

$$
1+s>p+q
$$

Inequality (1) is called the Grashof's criterion or Grashof's inequality. Paul [13] further proved that the Grashof's criterion (1) is both necessary and sufficient for the existence of at least one fully-rotatable link in a four-bar linkage. A linkage is called a Grashof linkage if it satisfies the Grashof's criterion, while it is called a non-Grashof linkage if the criterion is not satisfied. In addition, a linkage is called a change point mechanism or a Grashof neutral linkage if

$$
1+s=p+q
$$

At a change point, some instantaneous centers of the mechanism can not be uniquely determined [19], and the output behavior will become indeterminate.

Depending on the location of the shortest link, a Grashof linkage can be categorized into three different types:

(a) Crank-rocker mechanism: If the shortest link is adjacent to the ground, then the shortest link is fully rotatable, and the other ground-adjacent link will generate an oscillating motion.

(b) Double-crank mechanism: If the ground link is the shortest, both ground-adjacent links are fully rotatable.

(c) Double-rocker mechanism: If the coupler link is the shortest, both ground-adjacent links will be in oscillating motion, and the coupler is fully rotatable.

Thus it is known that the shortest link is always fully revolvable with respect to the other three links if the Grashof's criterion (1) is satisfied. The three movable links of a non-Grashof mechanism are always in oscillating motion, hence a non-Grashof linkage is also called a triple-rocker mechanism [13]. The non-Grashof double-rocker mechanism named by Grashof [7] is in fact a triple-rocker mechanism which satisfies inequal- ity (2). A classification of four-bar linkages is summarized in Table 1.

\section{STATIONARY AND UNCERTAINTY CONFIGURATIONS OF FOUR-LINK CHAINS}

A stationary configuration belongs to one class of the singular configurations of a kinematic chain, and so does an uncertainty configuration. A study of these configurations will provide a foundation to understand the mobility conditions of linkage mechanisms.

\section{Stationary configurations}

A stationary configuration of a kinematic chain is defined as the configuration when the joint of two adjacent links becomes temporarily inactive. Under such a condition, the two adjacent links are called the stationary links, and the corresponding joint is called the inactive joint $[11,19,20]$. At a stationary configuration, there is no relative motion between the two stationary links. One may regard the stationary links together with the inactive joint as a sole link [2], or a temporary rigid chain; and further, the instantaneous centers of a stationary link with respect to the other links will be coincident. For example, in Figure 1 (a), $\mathrm{I}_{13}$ and $\mathrm{I}_{34}$ are coincident, and so are $\mathrm{I}_{12}$ and $\mathrm{I}_{24}$. A mechanism will become locked if two adjacent stationary links are selected as the ground and the input link respectively; however, the input link can revolve continuously if it never becomes stationary. Figure 1 shows three different stationary configurations of a four-bar linkage, where link 2 is the input link and link 4 is the output link. Joint D in Figure 1(a), joint $\mathrm{C}$ in Figure 1 (b), and joint $A$ in Figure 1(c) are the inactive joints which lose their function at the corresponding configurations. In Figure 1(a), the inactive joint D coincides with the instant center I14, hence there is no relative motion existing between links 1 and 4 . It means that the output link is stationary with respect to the ground. In Figure 1(b), the inactive joint $\mathrm{C}$ coincides with the instant center $\mathrm{I}_{34}$, hence there is no relative motion existing between links 3 and 4 . Nevertheless, the input links in the above two cases are still movable.

Table 1. Classification of four-bar linkages $[13,14]$

\begin{tabular}{cccc}
\hline Case & $l+s \leq p+q$ & Shortest link & Type \\
\hline 1 & $<$ & Ground-adjacent & Crank-rocker \\
2 & $<$ & Ground & Double-crank \\
3 & $<$ & Coupler & Double-rocker \\
4 & $=$ & Any & Change point \\
5 & $>$ & Any & Triple-rocker \\
\hline
\end{tabular}


In Figure 1(c), the inactive joint A coincides with the instant center $\mathrm{I}_{12}$, hence there is no relative motion existing between links 1 and 2 . It means that the input link is stationary with respect to the ground and the mechanism is locked. In summary, at a stationary configuration of a four-link chain, if one of the stationary links is fixed temporarily, then the other stationary link will be in its limit position and become motionless at the moment. Since a link can not move in the same direction after reaching its limit position, it indicates the link can not make a full revolution. It is concluded that two links in a four-link chain can not revolve continuously with respect to each other if they are adjacent to an inactive joint at a stationary configuration. As shown in Figure 1, links 1 and 4 in Figure 1(a), links 3 and 4 in Figure 1(b), and links 1 and 2 in Figure 1(c), all become relatively motionless at the moment, and can not make a full revolution with respect to each other.

Figure 2 shows all the possible stationary configurations of a four-link chain, where links $k$ and $j$ are collinear, links $h$ and $i$ are the stationary links, and $\mathrm{I}_{h i}$ is the inactive joint. At the stationary configurations, the five instant centers $\left(\mathrm{I}_{h j}, \mathrm{I}_{h k}, \mathrm{I}_{i j}, \mathrm{I}_{i k}\right.$, and $\left.\mathrm{I}_{j k}\right)$ that do not

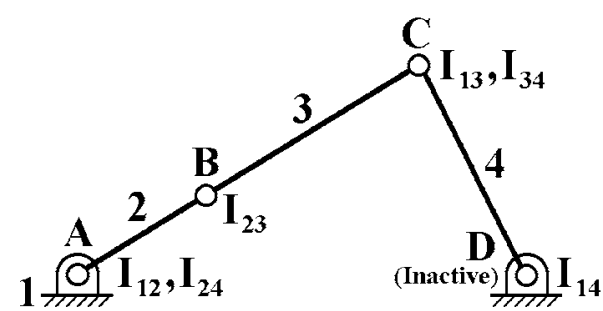

(a)

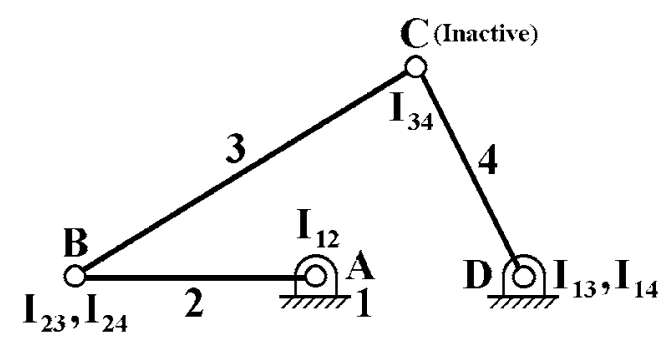

(b)

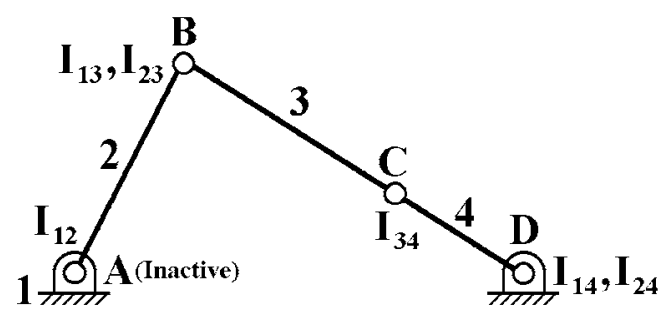

(c)

Fig. 1. Stationary configurations of four-bar linkages. include the inactive joint $\left(\mathrm{I}_{h i}\right)$ lie on the same line; and further, the instant centers $\mathrm{I}_{h k}$ and $\mathrm{I}_{i k}$ are coincident, and so are $\mathrm{I}_{h j}$ and $\mathrm{I}_{i j}$. The stationary configuration shown in Figure 2(a) is called the extended type, and the one shown in Figure 2(b) is called the folded type. As explained above, links $h$ and $i$ can not fully revolve with respect to each other.

\section{Uncertainty configurations}

In general, all links of a mechanism will undergo a constraint motion after being assembled; however, under some special circumstances, an uncertain state may exist such that a link can possess multiple motion paths. The uncertain state of a mechanism is called the change point, and the configuration is called the uncertainty configuration $[10,19]$. A mechanism possessing such a property is called a change point mechanism. The uncertainty configuration of a mechanism can be identified by verifying the rank equality of the Jacobian matrix and the extended Jacobian matrix of the motion constraint equations $[14,19]$. If the rank of the Jacobian matrix is less than that of the extended Jacobian matrix, it means the functional mapping between the primary and secondary coordinates of the mechanism is not unique. Physically, the angular velocity ratio between certain two links will be zero versus zero, which represents an uncertain state. By using the concept of the dead center configuration, Wu [19] introduced a systematic method of synthesizing the uncertainty configurations of mechanisms.

Figure 3(a) shows a four-bar linkage at an uncertainty configuration, whose links are collinear, and the instant centers $\mathrm{I}_{13}$ and $\mathrm{I}_{24}$ are indeterminate. The length of the two longer links is denoted by 1 , and that of the

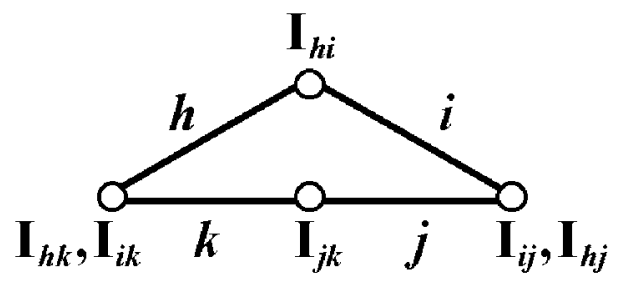

(a)

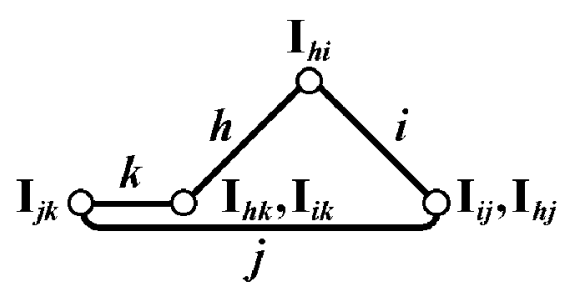

(b)

Fig. 2. All possible stationary configurations of a four-link chain. 
two shorter links is denoted by $s$. If the linkage is a parallelogram before reaching the uncertainty configuration as shown in Figure 3(b), the angular velocity ratio between links 4 and 2 being +1 , then the instant centers $\mathrm{I}_{13}$ and $\mathrm{I}_{24}$ are located at the infinity on the line. On the other hand, if the linkage is an anti-parallelogram before reaching the uncertainty configuration as shown in Figure 3(c), the angular velocity ratio between links 4 and 2 being -1 , then the instant centers $I_{13}$ and $I_{24}$ are located between the two instant centers $\mathrm{I}_{12}$ and $\mathrm{I}_{14}$, satisfying the relations $\mathrm{I}_{13} \mathrm{I}_{14}=(l-s) / 2$ and $\mathrm{I}_{12} \mathrm{I}_{24}=\mathrm{I}_{14} \mathrm{I}_{24}$ , respectively. From Figure 3, it is shown that the instant centers of two un-adjacent links can not be uniquely determined when the four links are collinear. Figure 4 shows all the fully-collinear configurations of four-link chains, where the instant centers $\mathrm{I}_{h i}, \mathrm{I}_{h k}, \mathrm{I}_{i j}$, and $\mathrm{I}_{j k}$ lie on the same line, while the locations of $\mathrm{I}_{h j}$ and $\mathrm{I}_{i k}$ are indeterminate. Among the figures, Figures 4(a) and 4 (b) are uncertainty configurations, while Figure 4(c) is an immovable configuration.

\section{LINK LENGTH RELATIONS AT STATIONARY AND UNCERTAINTY CONFIGURATIONS}

In the above section, it is shown that a four-link chain will become a triangular enclosed area at a stationary configuration, while all four links will become collinear at an uncertainty configuration. In this section we shall discuss the link-length relations of the fourlink chains when the above configurations are encountered.

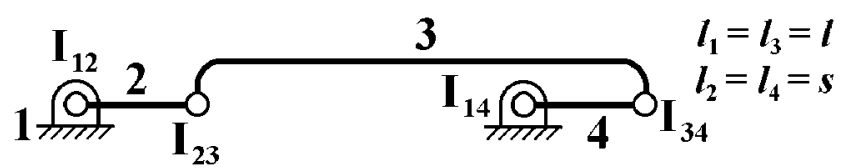

(a)

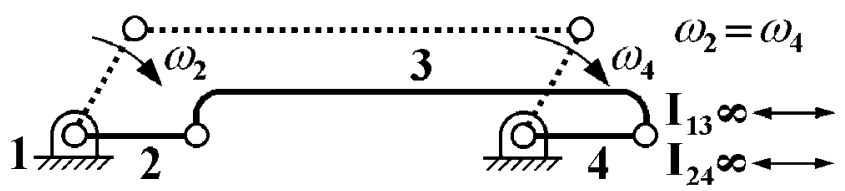

(b)

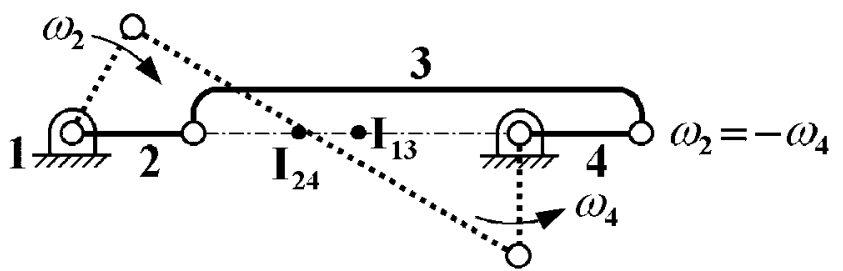

(c)

Fig. 3. An uncertainty configuration of a four-bar linkage.

\section{Stationary configurations}

Let the lengths of the three sides of a triangle formed by a four-link chain be $u, v$, and $w$. According to the triangle inequality, the following relations can be established.

$$
\begin{aligned}
& u<v+w \\
& v<u+w \\
& w<u+v
\end{aligned}
$$

Thus the link length relations of a four-link chain at a stationary configuration must satisfy inequalities (4), (5), and (6). When the four links with lengths $l, p$, $q$, and $s(1>p \geq q>s)$ are assembled into a triangle, the following four conditions can exist:

(a) length relation $l+s<p+q$ is valid.

(b) length relation $l+s>p+q$ is valid.

(c) neither of above length relations is valid.

(d) length relation results in an unrealizable configuration.

The four conditions are illustrated as follows:

\subsection{Length relation $l+s<p+q$ is valid}

Figure 5 shows two examples where the link length

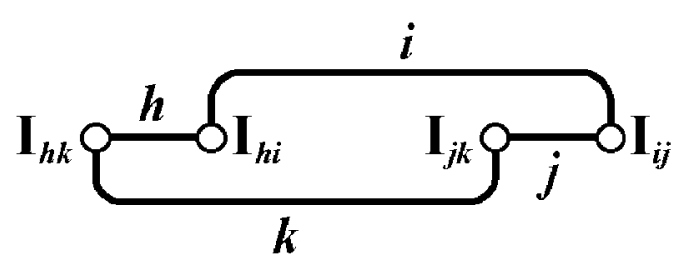

(a)

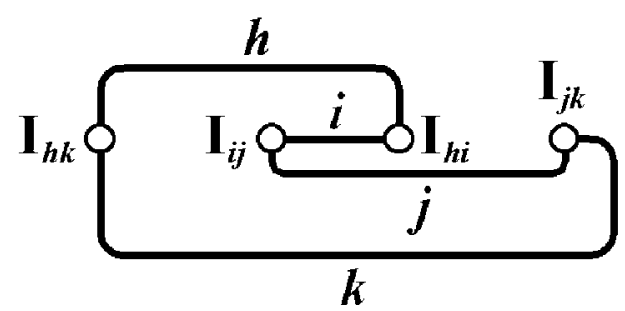

(b)

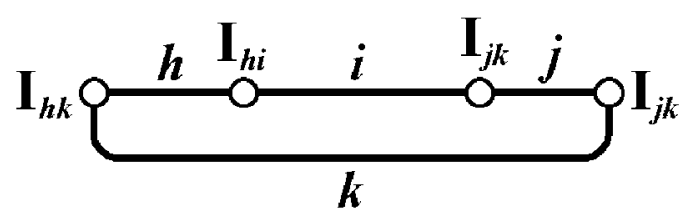

(c)

Fig. 4. All possible fully-collinear configurations of a four-link chain. 
relation $1+s<p+q$ can be found in such triangles. According to the triangle inequality, the link length of the four-link chain shown in Figure 5(a) must satisfy the relations:

$$
\begin{aligned}
& (l+s)<p+q \\
& p<(l+s)+q \\
& q<(l+s)+p
\end{aligned}
$$

Inequality (7) agrees with the link length relation of Grashof's criterion (Note that the equality form of the criterion is discussed later). Inequalities (8) and (9) are trivial since the length of any link must be less than the sum of the other three link lengths. In the same manner, the link-length of the four-link chain shown in Figure 5 (b) must satisfy the relations:

$$
\begin{aligned}
& (q-s)<1+p \\
& p<1+(q-s) \\
& 1<p+(q-s)
\end{aligned}
$$

Again, inequalities (10) and (11) are trivial relations. Inequality (12) agrees with the link length relation of Grashof's criterion.

\subsection{Length relation $l+s>p+q$ is valid}

Figure 6 shows two examples where the link length relation $l+s>p+q$ can be found in such triangles. According to the triangle inequality, the link-length of the four-link chain shown in Figure 6(a) must satisfy the relations:

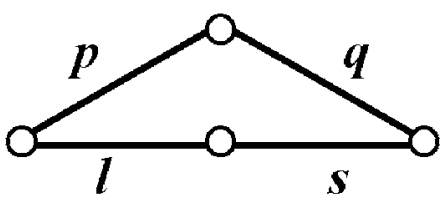

(a)

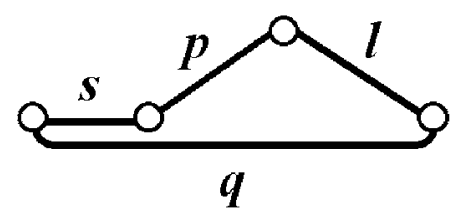

(b)

Fig. 5. Examples of stationary configurations whose length relations satisfy $l+s>p+q$.

$$
\begin{aligned}
& (p+q)<l+s \\
& s<l+(p+q) \\
& l<s+(p+q)
\end{aligned}
$$

Inequality (13) satisfies the relation $l+s>p+q$. Inequalities (14) and (15) are trivial relations. Similarly, the link lengths of the four-link chain shown in Figure 6 (b) must satisfy the relations:

$$
\begin{aligned}
& (l-q)<p+s \\
& s<(l-q)+p \\
& p<(l-q)+s
\end{aligned}
$$

Again, inequalities (16) and (17) are also trivial relations. Inequality (18) satisfies the relation $l+s>p$ $+q$.

\subsection{Neither of the above length relations is valid}

Figure 7 shows an example where neither the length relation $l+s<p+q$ nor $l+s>p+q$ can be found in such a triangle. According to the triangle inequality, the link lengths of the four-link chain shown in Figure

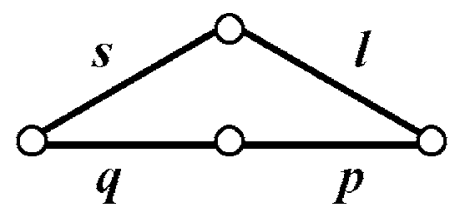

(a)

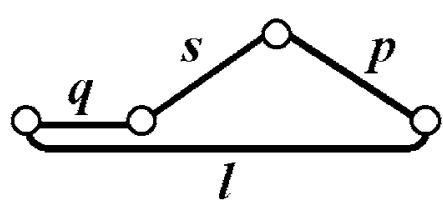

(b)

Fig. 6. Examples of stationary configurations whose length relations satisfy relation $l+s<p+q$.

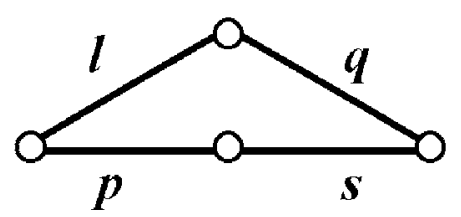

Fig. 7. An example of stationary configuration whose length relation does not satisfy $l+s<p+q$ or $l+s>p+q$. 
7 must satisfy the relations:

$$
\begin{aligned}
& (p+\mathrm{s})<l+q \\
& q<l+(p+\mathrm{s}) \\
& l<q+(p+\mathrm{s})
\end{aligned}
$$

Since $p<l$ and $s<q$, inequality (19) is trivial, and so are inequalities (20) and (21). In summary, it is shown that neither the relation $l+s\langle p+q$ nor $l+s\rangle$ $p+q$ exist in such a triangle.

\subsection{Length relation results in an unrealizable configuration}

Figure 8 shows two examples where the link length relations will result in unrealizable triangles. The fourlink chain shown in Figure 8(a) must satisfy the relation $l+q<p+s$. However, the configuration with the length relation can not exist since $l>p$ and $q>s$. The configuration shown in Figure 8(b) is also not realizable since $l>s$. Hence the four-link chain with the above length relations can not form such triangles.

\section{Uncertainty configurations}

At an uncertainty configuration the four links in a four-link chain will become collinear. As the four links with lengths $l, p, q$, and $\mathrm{s}(l>p \geq q>s)$ are assembled into a closure with zero area, the following conditions may occur:

(a) length relation $l+s=p+q$ is valid.

(b) length relation results in an unrealizable zero area closure.

The two conditions are illustrated as follows:

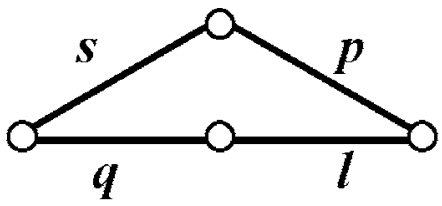

(a)

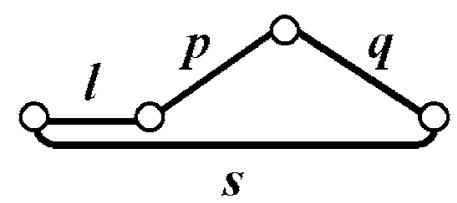

(b)

Fig. 8. Examples of stationary configurations whose length relations result in unrealizable configurations.

\subsection{Length relation $l+s=p+q$ is valid}

Figure 9 shows two examples of the uncertainty configurations satisfying the length relation $l+s=p+$ $q$. In Figure 9(a), the distance between the two endpoints $A$ and $B$ is

$$
A B=l+s=p+q
$$

Eq. (22) satisfies the length relation $l+p=q+s$. Similarly, in Figure 9(b), the distance between two endpoints $A$ and $B$ is

$$
A B=l=p+q-s
$$

Thus Eq. (23) also satisfies the length relation $l+$ $s=p+q$.

\subsection{Length relation results in an unrealizable zero area closure}

Figure 10 shows three examples that the length relations will result in unrealizable zero area closures. The configuration shown in Figure 10(a) must satisfy the relation $l+p=q+s$. However, the configuration with the length relation can not exist since $l>q$ and $p<$ $s$. The configurations shown in Figures $10(\mathrm{~b})$ and (c) must satisfy the relations $l+q=p+s$ and $l+s=p+q$, respectively. However, the two configurations also can not exist since $l$ is not the shortest link length. Thus the above configurations are physically unrealizable. Three types of link length relations resulting in unrealizable uncertainty configurations are summarized as follows: (a) $l+p=q+s$ is satisfied, while $l>p \geq q>s$ is violated.

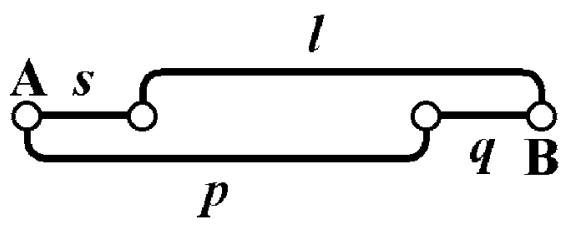

(a)

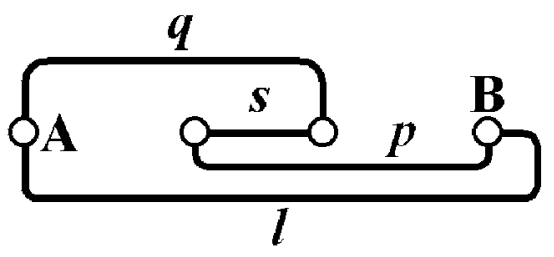

(b)

Fig. 9. Examples of the uncertainty configurations whose length relations satisfy $l+s=p+q$. 
(b) $l+q=p+s$ is satisfied, while $l>p \geq q>s$ is violated. (c) $l+\mathrm{s}=p+q$ is satisfied, while $l>p \geq q>s$ is violated.

Note that the above length relations may still be valid if the constraints $l>p \geq q>s$ are modified. This issue is to be discussed later.

\section{PROOF OF GRASHOF'S THEOREM}

Figure 11 shows all the link permutations of a four-link chain having link-lengths $l, p, q$, and $s$. Among them, the kinematic chains in Figures 11(a) and 11(b), 11(c) and 11(d), 11(e) and 11(f) are topologically identical. Thus only the chains in Figures 11(a), 11(c), and 11(e) will be considered. In this paper, the four-link chains in Figures 11(a), 11(c), and 11(e) are called Type I (ordered by $s-p-l-q$ ), Type II (ordered by $s-l-$ $p-q$ ), and Type III (ordered by $s-l-q-p$ ), respectively.

\section{Proof of Grashof's criterion}

In this section, Grashof's criterion is proved by first listing all the stationary configurations occurring in a four-link chain, and then establishing the valid length relations based on the triangle inequality. Tables 2, 3, and 4 list the link length arrangements of Types I,

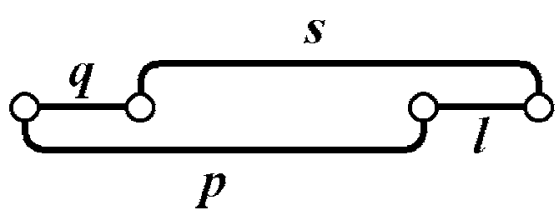

(a)

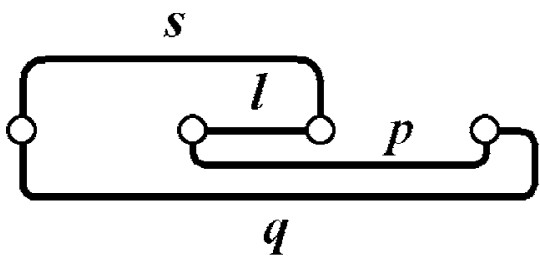

(b)

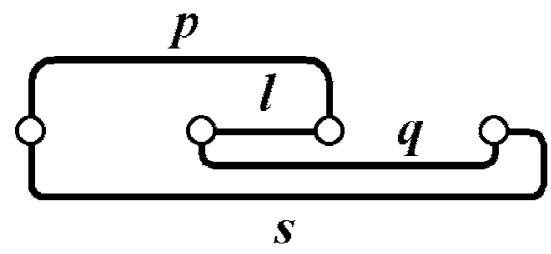

(c)

Fig. 10. Examples of uncertainty configurations whose length relations result in unrealizable configurations.
II, and III four-link chains at their stationary configurations, respectively. Each table contains two groups: (a) the shortest link belongs to one of the collinear links, and (b) the shortest link belongs to one of the stationary links. In cases $3,4,7$, and 8 of Table 2 , the shortest link belongs to one of the collinear links. The length relation can be derived from case 8 using the triangle inequality, as illustrated in subsection 1.1 of above section. The length relation is trivial in case 3 since $s<q$ and $p<l$ are always true. Similarly, trivial length relations can be found in case 4 . The length relation is unrealizable in case 7 since $l<q+(s-p)$ is always false. In cases $1,2,5$, and 6 of Table 2 , the shortest link belongs to one of the stationary links. The length relation $l+s>p+q$ can be derived from case 5 using the triangle inequality, as illustrated in subsection 1.2 of above section. The unrealizable length relations can be derived in cases 1,2, and 6 when one follows the same logic as that in case 7 . As a summary from Table 2 , it can be shown that when the length relation $l+s<$ $p+q$ is true (case 8 ), the shortest link must belong to one of the collinear links at a stationary configuration; and the shortest link can make a full revolution with respect to the adjacent collinear link since it will never become a stationary link during the complete cycle of the motion. On the other hand, when the length relation $l+s>p+$

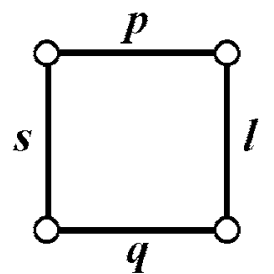

(a)

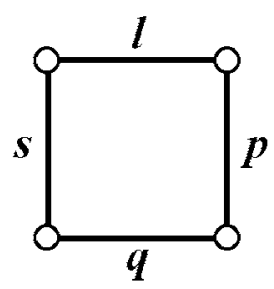

(c)

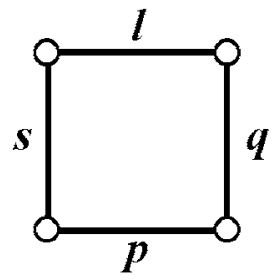

(e)

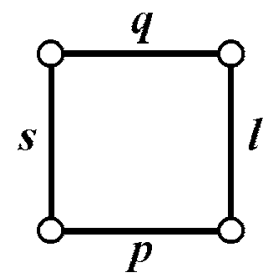

(b)

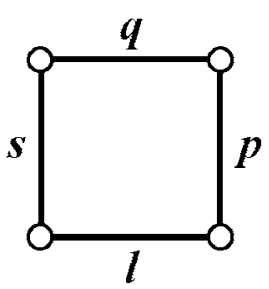

(d)

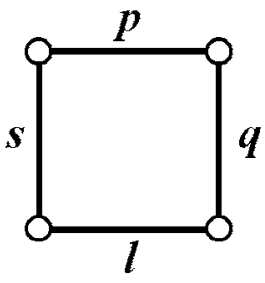

(f)
Fig. 11. All possible link permutations of a four-link chain. 
$q$ is true (case 5), the shortest link must belong to one of the stationary links at a stationary configuration; and the shortest link can not make a full revolution. Accordingly, the same conclusions can be derived from Table 3 and Table 4 for Type II and Type III four-link chains, respectively. Finally, when the length relation $l+s=p+q$ is satisfied, the shortest link is collinear with the other three links at a singular configuration; if the shortest link is an input link, it can revolve continuously with respect to the adjacent link without any problem,

\section{Table 2. Stationary configurations of Type I four-link chains}

\begin{tabular}{|c|c|c|c|}
\hline Case & Sketch & Stationary links & Length relation \\
\hline 1 & & $p$ and $s$ & Unrealizable \\
\hline 2 & & $q$ and $s$ & Unrealizable \\
\hline 3 & & $l$ and $q$ & Trivial \\
\hline 4 & & $l$ and $p$ & Trivial \\
\hline 5 & & $p$ and $s$ & $1+s>p+q$ \\
\hline 6 & & $q$ and $s$ & Unrealizable \\
\hline 7 & & $l$ and $q$ & Unrealizablc \\
\hline 8 & 4 & $l$ and $p$ & $1+s<p+q$ \\
\hline
\end{tabular}

Table 3. Stationary configurations of Type II four-link chains

\begin{tabular}{|c|c|c|c|}
\hline Case & Sketch & Stationary links & Length relation \\
\hline 1 & & $l$ and $s$ & $l+s>p+q$ \\
\hline 2 & & $q$ and $s$ & Unrealizable \\
\hline 3 & & $p$ and $q$ & $l+s<p+q$ \\
\hline 4 & & $l$ and $p$ & Trivial \\
\hline 5 & & $l$ and $s$ & Unrealizable \\
\hline 6 & & $q$ and $s$ & $l+s>p+q$ \\
\hline 7 & & $p$ and $q$ & Unrealizable \\
\hline 8 & $q$ & $l$ and $p$ & $l+s<p+q$ \\
\hline
\end{tabular}

but if the shortest link is not an input link, its motion path will be indeterminate at the collinear configuration. Nevertheless, the relative motion between the shortest link and its adjacent link will be a full revolution.

From the above deductions, two cases can be established as follows:

Case 1: If and only if the length relation $l+s \leq p+q$ is true, the shortest link must be one of the collinear links at a stationary configuration, and can fully revolve with respect to the adjacent collinear link.

Case 2: If and only if the length relation $l+s>p+q$ is true, the shortest link must be one of the stationary links at a stationary configuration, and can not fully revolve with respect to the adjacent

Table 4. Stationary configurations of Type III four-link chains

\begin{tabular}{|c|c|c|c|}
\hline Case & Sketch & Stationary links & Length relation \\
\hline 1 & & $l$ and $s$ & $l+s>p+q$ \\
\hline 2 & & $p$ and $s$ & Unrealizable \\
\hline 3 & & $p$ and $q$ & $l+s<p+q$ \\
\hline 4 & & $l$ and $q$ & Trivial \\
\hline 5 & & $l$ and $s$ & Unrealizable \\
\hline 6 & t & $p$ and $s$ & $l+s>p+q$ \\
\hline 7 & & $p$ and $q$ & Unrealizable \\
\hline 8 & $p$ & $l$ and $q$ & $l+s<p+q$ \\
\hline
\end{tabular}

Table 5. Uncertainty configurations of Type I four-link chains

\begin{tabular}{|c|c|c|c|}
\hline Case & Sketch & Length relation & Remarks \\
\hline 1 & $\underbrace{5}_{4}$ & $l+q=p+s$ & $\begin{array}{c}\text { Unrealizable for } \\
\text { violating } l>p \geq q>s\end{array}$ \\
\hline 2 & $\underbrace{4}_{1} \overbrace{p}^{s}$ & $l+p=q+s$ & $\begin{array}{c}\text { Unrealizable for } \\
\text { violating } l>p \geq q>s\end{array}$ \\
\hline 3 & $\underbrace{\frac{p}{0}, ~}_{q}$ & $l+s=p+q$ & $\begin{array}{c}\text { Unrealizable for } \\
\text { violating } l>p \geq q>s\end{array}$ \\
\hline 4 & $\stackrel{4}{\circ}$ & $l+s=p+q$ & Valid \\
\hline 5 & $\frac{14}{n}$ & $1+s=p+q$ & $\begin{array}{c}\text { Unrealizable for } \\
\text { violating } l>p \geq q>s\end{array}$ \\
\hline 6 & $\stackrel{p}{1}$ & $l+s=p+q$ & $\begin{array}{c}\text { Unrealizable for } \\
\text { violating } l>p \geq q>s\end{array}$ \\
\hline
\end{tabular}


Table 6. Uncertainty configurations of Type II four-link chains

\begin{tabular}{|c|c|c|c|}
\hline Case & Sketch & Length relation & Remarks \\
\hline l & $\underbrace{}_{q} \overbrace{q}$ & $l+s=p+q$ & Valid \\
\hline 2 & $\underbrace{4}_{p}$ & $l+p=q+s$ & $\begin{array}{c}\text { Unrealizable for } \\
\text { violating } l>p \geq q>s\end{array}$ \\
\hline 3 & $\left\{\begin{array}{l}\underbrace{}_{4} p \\
0\end{array}\right.$ & $l+q=p+s$ & $\begin{array}{c}\text { Unrealizable for } \\
\text { violating } l>p \geq q>s\end{array}$ \\
\hline 4 & $\left\{\begin{array}{lll}4 & 5\end{array}\right.$ & $l+q=p+s$ & $\begin{array}{c}\text { Unrealizable for } \\
\text { violating } l>p \geq q>s\end{array}$ \\
\hline 5 & ת: & $I+q=p+s$ & $\begin{array}{c}\text { Unrealizable for } \\
\text { violating } l>p \geq q>s\end{array}$ \\
\hline 6 & $\stackrel{1}{=}$ & $l+q=p+s$ & $\begin{array}{c}\text { Unrealizable for } \\
\text { violating } l>p \geq q>s\end{array}$ \\
\hline
\end{tabular}

stationary link.

A lemma proved in Paul's paper [13] stated that: a link in a four bar chain can revolve relative to another link if, and only if, some link revolves relative to an adjacent link. By adding the lemma to the above deductions, the two cases can be rephrased as follows:

If and only if the length relation satisfies $l+s \leq p$ $+q$ in a four-link chain, the shortest link can always fully revolve with respect to the other links; If and only if the length relation satisfies $l+s>p+q$, none of the links can revolve continuously with respect to the other links.

Thus the proof of Grashof's criterion is completed.

\section{Proof of change point condition}

It is shown that at an uncertainty configuration of a four-link chain, the four links will become collinear and the instant centers of any two un-adjacent links can not be uniquely determined. Such a four-link chain will become a change point mechanism when a ground link is chosen. Tables 5, 6, and 7 list the link length arrangements of Types I, II, and III four-link chains at their uncertainty configurations, respectively. In Table 5 , the length relation $l+s=p+q$ can be derived in case 4 , while the length relations are unrealizable in the remaining five cases. In Tables 6 and 7, the length relation $l+s=p+q$ can be derived in case 1 , while the length relations are unrealizable in the other ten cases. Summarized from Tables 5, 6, and 7, a conclusion can be made as follows:

The uncertainty configuration of a four-link chain will occur if, and only if, the length relation satisfies $l$ $+s=p+q$.

As mentioned above, a four-link chain possessing an uncertain configuration will become a change point
Table 7. Uncertainty configurations of Type III four-link chains

\begin{tabular}{|c|c|c|c|}
\hline Case & Skctch & Length relation & Remarks \\
\hline 1 & $8 \underbrace{s}_{p} \overbrace{q}$ & $l+s=p+q$ & Valid \\
\hline 2 & $\underbrace{p}_{q}$ & $l+q=p+s$ & $\begin{array}{c}\text { Unrealizable for } \\
\text { violating } l>p \geq q>s\end{array}$ \\
\hline 3 & $\left\{\begin{array}{l}s \\
\qquad \underbrace{}_{n}\end{array}\right.$ & $l+p=q+s$ & $\begin{array}{c}\text { Unrealizable for } \\
\text { violating } l>p \geq q>s\end{array}$ \\
\hline 4 & 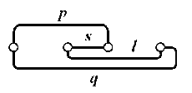 & $l+p=q+s$ & $\begin{array}{c}\text { Unrealizable for } \\
\text { violating } l>p \geq q>s\end{array}$ \\
\hline 5 & $\stackrel{4}{\stackrel{4}{\prime}}$ & $l+p=q+s$ & $\begin{array}{c}\text { Unrealizable for } \\
\text { violating } l>p \geq q>s\end{array}$ \\
\hline 6 & $\stackrel{4}{\longrightarrow}$ & $l+p=q+s$ & $\begin{array}{c}\text { Unrealizable for } \\
\text { violating } l>p \geq q>s\end{array}$ \\
\hline
\end{tabular}

mechanism when a ground link is chosen. The above conclusion can be rephrased as follows:

A four-link chain will become a change point mechanism after the process of the kinematic inversion if, and only if, the length relation satisfies $l+s=p+q$.

There are some special cases that the change point conditions occur in a four-link chain while the constraints $l>p \geq q>s$ are violated. Cases 3, 5, and 6 in Table 5 satisfy $l+p=q+s$ while violating $l>p \geq q>$ $s$; Case 2 in Tables 5 and 6 , and cases 3 through 6 in Table 7 satisfy $l+p=q+s$ while violating $l>p \geq q>$ $s$. The only reasonable solution to the above cases is that the link lengths satisfy $l=p=q=s$. A mechanism satisfied such condition is called a triple change point mechanism by Barker [3].

Furthermore, case 1 in Table 5, cases 3 through 6 in Table 6 , and case 2 in Table 7 satisfy $l+q=p+s$ while violating $l>p \geq q>s$. The above cases will be valid if $l=p \geq q=s$ or $l=p=q=s$ is satisfied. When the lengths satisfy $l=p \geq q=s$, if the order of the link lengths is $l$ $-s-l-s$, then the linkage is a parallelogram or an antiparallelogram (a bowtie); if the order of the link lengths is $l-l-s-s$, then the linkage is a kite type. When the lengths satisfy $l=p=q=s$, the linkage is a triple change point linkage.

\section{CONCLUSION}

In this paper, Grashof's theorem or the mobility condition of planar four-bar linkages is justified from the perspective of the occurrence of singular configurations of four-link chains. It is shown that a four-link chain will become a triangular enclosed area at a stationary configuration, while all four links will become collinear at an uncertainty configuration. By permuting the link lengths in the stationary configurations of four- 
link chains, all cases of stationary configurations are generated and categorized in Tables 2, 3, and 4. Likewise, all cases of uncertainty configurations are generated and categorized in Tables 5, 6, and 7. An examination of the triangle inequality on all cases of Tables 2, 3, and 4 has shown that, after excluding the trivial cases and unrealizable cases, the valid cases will lead to the same conclusions as Grashof's theorem. An examination of the length equality between two end points on the cases of Tables 5, 6, and 7 has shown that, after excluding the trivial cases and unrealizable cases, the valid cases will lead to the change point condition.

\section{NOMENCLATURE}

$\begin{array}{ll}A, B, C, D & \begin{array}{l}\text { revolute joints } \\ \text { instant center of links labeled } m \text { and } n\end{array} \\ I_{m n} & \begin{array}{l}\text { longest link length of a four-link chain } \\ l\end{array} \\ l_{n} & \begin{array}{l}\text { length of a link labeled } n \\ \text { second longest link length of a four-link } \\ \text { chain }\end{array} \\ q & \text { second shortest link length of a four-link } \\ \text { chain } & \text { shortest link length of a four-link chain } \\ s & \text { side lengths of a triangle }\end{array}$

\section{REFERENCES}

1. Angeles, J. and Callejas, M., “An Algebraic Formulation of Grashof's Mobility Criteria with Application to Linkage Optimization Using Gradient-Dependent Methods," J. Mech. Transm-T. ASME, Vol. 106, No. 3, pp. 327-332 (1984).

2. Baker, J.E., "Limit Positions of Spatial Linkages via Connectivity Sum Reduction," J. Mech. Des. -T. ASME, Vol. 101, No. 3, pp. 504-508 (1979).

3. Barker, C.R., "A Complete Classification of Planar Four-Bar Linkages," Mech. Mach. Theory, Vol. 20, No. 6, pp. 535-554 (1985).

4. Blaschke, L. and Muller, H.R., Ebene Kinematik, Oldenbourg, Munchen, pp. 85-86 (1956).

5. Cohan, S.M. and Yang, D.C.H., "Mobility Analysis of Planar Four-Bar Mechanisms through the Parallel Coor- dinate System," Mech. Mach. Theory, Vol. 21, No. 1, pp. 63-71 (1986).

6. Freudenstein, F., “Approximate Synthesis of Four-Bar Linkages," Trans. ASME, Vol. 77, pp. 853-861 (1955).

7. Grashof, F., Theoretische Mashinenlehre, Leipzig, pp. 113-118 (1883).

8. Harding, B.L., "Derivation of Grashof's Law," Am. Soc. Eng. Edu. Mach. Des. Bull., pp. 1-10 (1962).

9. Hartenberg, R.S. and Denavit, J., Kinematic Synthesis of Linkages, McGraw-Hill, New York, pp. 75-78 (1964).

10. Hunt, K.H., Kinematic Geometry of Mechanisms, Oxford University Press, Oxford (1978).

11. Kiang, Y.C., "Stationary Configurations of Eight-Bar Kinematic Chains," Master Thesis, Department of Mechanical Engineering, National Cheng Kung University, Tainan, Taiwan (1991).

12. Midha, A., Zhao, Z.L., and Her, I., "Mobility Conditions for Planar Linkages Using Triangle Inequality and Graphical Interpretation," J. Mech. Transm.-T. ASME, Vol. 107, No. 3, pp. 394-400 (1985).

13. Paul, B., "A Reassessment of Grashof's Criterion," J. Mech. Des.-T. ASME, Vol. 101, No. 3, pp. 515-518 (1979).

14. Paul, B., Kinematics and Dynamics of Planar Machinery, Prentice-Hall, Englewood Cliffs, NJ (1979).

15. Ting, K.L., "Five-bar Grashof Criteria," J. Mech. TransmT. ASME, Vol. 108, No. 4, pp. 533-537 (1986).

16. Ting, K.L., "Mobility Criteria of Single-loop N-Bar Linkages," J. Mech. Transm-T. ASME, Vol. 111, No. 4, pp. 504-507 (1989).

17. Ting, K.L. and Liu, Y.W., "Rotatability Laws for N-Bar Kinematic Chains and Their Proof," J. Mech. Des.-T. ASME, Vol. 113, No. 1, pp. 32-39 (1991).

18. Williams, R.L. II and Reinholtz, C. F., "Proof of Grashof's Law Using Polynomial Discriminants," J. Mech. TransmT. ASME, Vol. 108, No. 4, pp. 562-564 (1986).

19. Wu, L.I., "On the Singular Configurations of Planar Linkage Mechanisms," Ph.D. Dissertation, Department of Mechanical Engineering, National Cheng Kung University, Tainan, Taiwan (1987).

20. Yan, H.S. and Wu, L.I., "Stationary Configurations of Planar Six-Bar Kinematic Chains," Mech. Mach. Theory, Vol. 23, No. 4, pp. 287-293 (1988). 